Electrolyse das von F. Kohlrausch aufgestellte Gesetz der unabbängigen Wanderung.

2. Die Beweglichkeit $u 10^{7}$ des Lithiums ist 21 .

3. In Beziehung auf die Beweglichkeit der Jonen müssen, im Gegensatz zur Meinung von Kohlrausch, die kohlensauren Verbindungen von den schwefelsauren Verbindungen getrennt werden. Die Beweglichkeiten der Jonen $\mathrm{K}$ und $\mathrm{Na}$ sind in den Carbonaten dieselben, wie in den Electrolyten, die aus zwei einwerthigen Elementen bestehen.

4. Die Beweglichkeit $v 10^{7}$ der Gruppe $\mathrm{CO}_{3}$ ist 29.

5. In den Verbindungen $\mathrm{Li}_{2} \mathrm{CO}_{3}$ und $\mathrm{Li}_{2} \mathrm{SO}_{4}$ wird die Leitungsfähigkeit durch besondere Verhältnisse beeintlusst.

\title{
VII. Ueber das galvanische Verhalten der Kohle ${ }^{1}$; von Hanichi Muraoka aus Japan.
}

\section{$\S 1$.}

Es ist durch die in den letzten Monaten erschienenen Arbeiten von Siemens ${ }^{2}$, Beetz ${ }^{3}$ ) und Borgmann ${ }^{4}$ ) als sicher nachgewiesen worden, dass die Kohle bei höherer Temperatur den electrischen Strom besser leitet, als bei niederer, und dass die entgegengesetzten Resultate von $A$ uerbach${ }^{5}$ ) auf Contactfehlern beruhen. Ich habe vor dem Erscheinen der Siemens'schen Arbeit eine Reihe von verschiedenen Kohlen auf ihr galvanisches Leitungsvermögen untersucht und habe für alle Kohlensorten das gleiche Resultat wie die oben genannten erhalten.. $\mathrm{Da}$ jedoch meine Untersuchungsmethode in Beziehung auf Contacte, auf die es bei diesen Untersuchungen vor allen Dingen ankommt,

1) Auszug aus der Dissertation. Strassburg 1881.

2) Siemens, Berl. Ber. 1880. p. 1 .

3) Beetz, Wied. Ann. 12. p. 65. 1881.

4) Borgmann, Wied. Ann. 11. p. 1041. 1880.

5) Auerbach, Gött. Nachr. p. 269. 1879. 
und auch in anderen Punkten eine von der Siemens'schen abweichende ist, da ich ferner eine grosse Anzahl von verschiedenen Kohlensorten untersuchte, so will ich kurz meine Methode und Resultate angeben. Ausserdem habe ich gefunden, dass zwischen verschiedenen Kohlensorten Thermoströme von ziemlich bedeutender Stärke entstehen, und habe bei einer Anzahl ron Combinationen verschiedener Kohlen deren Grösse gemessen.

\section{$\S$.. Contacte, Heizung und Messmethode.}

Was zunächst die Contacte anbetrifft, so gibt ein einfaches Anlöthen der Drähte mit Zinnloth oder Schlagloth an die verkupferten Kohlenenden wohl bis $100^{\circ}$ sichere Berührung, bei höheren Temperaturen aber halten diese Contacte nicht mehr aus. Die Siemens'sche Methode, Kohle und Drähte zusammen zu verkupfern, konnte ich bei den mir $z u$ Gebote stehenden Mitteln nicht anwenden, da es sehı schwer ist, eine gute Verkupferung der Kohle und Drähte zusammen zu erlangen. Ich benutzte endlich die auffallend geringe Ausdehnung der Kohle, uber welche ich

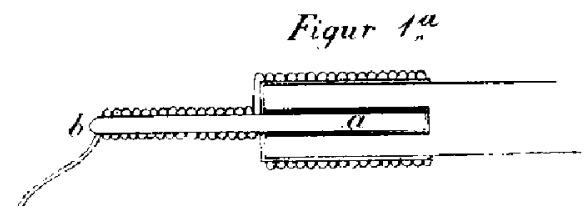

$$
\text { Figur to }
$$

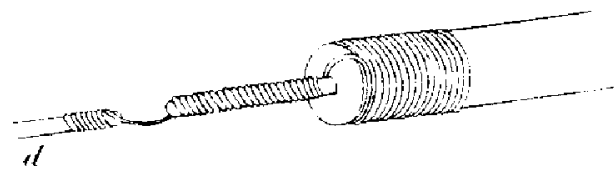

weiter unten einige Messungen angeben werde, dazu, um auf bequeme Weise Contacte zu erhalten, die allen Anforderungen genügen.

Ich bohrte nämlich, wie die Fig. $1 a$ zeigt, in die Enden der Kohle axial Löcher a von 10 bis $15 \mathrm{~mm}$ Länge und verkupterte die Enden der Kohle und die Löcher. In diese verkupferten Löcher brachte ich Silberstückchen oder auch Schlagloth hinein und steckte dann einen dicht anschliessenden Kupferstab $b$ hinzu, dessen Ende noch aus der Kolle etwa $20 \mathrm{~mm}$ herausragte. An dieses heraustretende 
Ende des Kupferstabes sowohl als auch an das rerkupferte Ende der Kohle wurden dünne Schlaglothplatten gelegt. Darauf wurde ein dünnerer Draht fest und dicht an die Kohle und den Kupferstab gewickelt (Fig. 1b) und nun das Ganze unter Benutzung von Borax in der Stichflamme erhitzt, bis das Schlagloth schmolz. Dabei wurde der Kupferstab in das Loch der Kohle hineingedrückt, bis er das Ende desselben erreichte. Die Folge davon war, dass der Kupferstab und die inneren Wände des Loches in der Kohle, die ja verkupfert waren, in vollständig metallischem Contact standen. Dieser Contact nun, der für niedere Temperaturen ebenso sicher ist wie der von Siemens, muss bei höheren Temperaturen mindestens ebenso gut bleiben. Der Kupferstab im Inneren der Kohle dehnt sich mit steigender Temperatur weit stärker aus, als die Kohle, und der Contact kann deshalb niemals gelockert werden.

Um die Kohle auf hohe Temperaturen zu bringen und sie eine Zeit lang (15-20 Minuten) auf bestimmter Temperatur $z u$ erhalten, brachte ich dieselbe in einen Heizapparat, der folgendermassen construirt war. Eine Eisenröhre von $350 \mathrm{~mm}$ Länge und $40 \mathrm{~mm}$ Durchmesser, die auf vier Eisenfüssen steht, enthält im. Inneren eine längere und engere Eisenröhre von $450 \mathrm{~mm}$ Länge und $20 \mathrm{~mm}$ Durchmesser, die durch verschiebbare Eisenringe in ihr befestigt ist. Die innere Röhre diente zur Aufnahme einer Glasröhre, welche die Kohle und ein Thermometer enthielt. Alle Zwischenräume wurden mit Asbest ganz dicht zugestopft. Unter dem ganzen Röhrensystem befanden sich eine Reihe Brenner. Die ganze Vorrichtung wurde mit einer Eisenhülle gedeckt, welche mit Fenstern versehen war, um den Luftzug und die Regulirung der Brenner zu gestatten. Die Brenner konnten leicht so regulirt werden, dass die Temperatur 15-20 Minuten lang constant blieb oder höchstens um $5^{0}$ schwankte. Man konnte sicher sein, dass in dieser Zeit die Kohle durchweg die Temperatur angenommen hatte, die das Thermometer zeigte.

Zur Widerstandsmessung bediente ich mich der Methode der Wheatstone'schen Brücke. Da die Verbindungsdrähte, 
welche theilweise in dem Heizapparate waren, eine höhere Temperatur und infolge dessen grössere Widerstände bekamen, dieser Einfluss sich aber durch Rechnung schwer genau corrigiren liess, so hatte ich diese Verbindungsdrähte aus dicken Kupferstäben gemacht, deren Widerstände, an sich klein, auch durch Erwärmung keinen Einfluss auf das Resultat hervorbrachten. Diese dicken Kupferstäbe waren an die Kupferstäbe $b$ (Fig. $1 b$ ) mit Silber angelöthet.

\section{§ 3. Beobachtungen und Resultate.}

Im Folgenden werden die Resultate der Widerstandsmessungen an rerschiedenen Koblen $(\mathrm{I}-\mathrm{X})$ angegeben. Um diese Kohlen etwas bestimmter zu charakterisiren, babe ich ihr specifisches Gewicht, ihren Ausdehnungscoëfficienten und theilweise ihren Gehalt an Kohlenstoff bestimmt. - Die Messung der Ausdehnungscoëfficienten geschah mit einem von Edelmann ${ }^{1}$ ) construirten Apparate, bei welchem die Verlängerung des zu untersuchenden Stabes durch den Ausschlag eines mit Spiegel versehenen Winkelhebels gemessen wird. - Die angegebenen Zahlen für die Widerstände sind ausgedrückt in Siemenseinheiten, und jede ist das Mittel aus einer Reihe von Beobachtungen. Es ergab sich, dass die Widerstandszunahme nicht proportional der Temperaturabnahme ist; es wurde daher aus jeder Beobachtungsreibe eine quadratische Formel berechnet:

$$
W_{t}=W_{0}\left(1+\alpha t+\beta t^{2}\right)
$$

in welcher $W_{t}$ den bei der Temperatur $t$ beobachteten Widerstand der betreffenden Kohle, $\boldsymbol{W}_{0}$ denselben bei $0^{\circ}$ bedeutet, und $\alpha$ und $\beta$ Constanten sind. - Es ergab sich, dass durch eine solche Formel die Beobachtungen hinreichend genau dargestellt werden konnten.

Aus jedem $W_{0}$ ist sodann mit Berücksichtigung der gemessenen linearen Dimensionen der Kohlenstücke für jedes derselben der specifische Widerstand berechnet, d. h. derjenige, welche ein Meter der Kohle von 1 qmm Querschnitt bei der Temperatur $0^{\circ}$ in S.-E. haben würde.

1) Edelmann, Neuere Apparate für naturw. Schule. p. 124. 1879. 
Kohle I.

Sibirischer Graphit aus dem Berliner mineralogischen Museum. Es wurden parallelepipedische Stücke aus einem grösseren Stücke mittelst einer Kreissäge geschnitten.

Specifisches Gewicht . . . . . 1.8

Procentgehalt an Kohlenstoff . . . 98,0

Ausdehnungscoëfficient . . . . . 0,0000038

$\begin{array}{lcccc}\text { Temperatur } & 26 & 194 & 251 & 302 \\ W_{l} \text { beobachtet } & 0,0785 & 0,0693 & 0,0665 & 0,0646\end{array}$

$W_{t}=0,0799\left(1-0,000739 t+0,000000273 t^{2}\right)$.

Specifischer Widerstand $=12,20$.

Kohle II.

Pariser Gasretortenkohle (Elementenkohle).

Specifisches Gewicht . . . 1,80

Ausdehnungscoëfficient . . . . 0,000 0015

Temperatur $\quad 17,5 \quad 100$

$W_{t}$ beobachtet $\quad 0,246 \quad 0,240$

$\left.W_{t}=0,247(1-0,00030 t) \cdot{ }^{1}\right)$

Specifischer Widerstand $=52,23$.

Kohle III.

Künstliche Kohle, wie sie für electrisches Licht benutzt wird.

$$
\begin{array}{lcc}
\text { Temperatur } & 17,0 & 100 \\
W_{t} \text { beobachtet } & 0,360 & 0,348 \\
W_{t}=0,362(1 & -0,000 & 405 t)
\end{array}
$$

Specifischer Widerstand $=48,44$.

$$
\text { Kohle IV. }
$$

Eine zweite künstliche Kohle für electrisches Licht.

Specifisches Gewicht . . . . . 2,37

Ausdehnungscoëfficient . . . 0,000001 5 .

$\begin{array}{lllll}\text { Temperatur } & 26 & 165 & 213 & 335\end{array}$

$W_{t}$ beobachtet $\quad 0,228 \quad 0,219 \quad 0,217 \quad 0,211$

$$
W_{t}=0,230\left(1-0,000425 t+0,000000715 t^{2}\right) \text {. }
$$

Specifischer. Widerstand $=42,90$.

1) Für diejenigen Kohlen, deren Widerstandsänderungen nur bis $100^{\circ}$ untersucht sind, wurde die lineare Formel angewendet und nur $\alpha$ berechnet. 


\section{Eoble V.}

Künstliche Kohle von Coaks für electrisches Licht, bezogen aus der Maschinenfabrik von Heilmann \& Comp. in Mühlhausen.

Specifisches Gewicht . . . . . . 1,90

Procentgehalt an Kohlenstoff . . . 98,1

Ausdebuungseoëfficient. . . . . . 0,000000 32

$\begin{array}{lllll}\text { Temperatur } & 28 & 165 & 240 & 338\end{array}$

$W$, beobachtet $\quad 0,139 \quad 0,135 \quad 0,134 \quad 0,132$

$\boldsymbol{W}_{t}^{+}=0,140\left(1-0,000156 t+0,0000000524 t^{2}\right)$.

Specifischer Widerstand $=39,10$.

Kohle VII. ${ }^{1}$ )

Bleistift ron M. Faber.

Specifisches Gewicht . . . . . . 236

Procentgehalt an Kohlenstuff' , . . 52,0

Ausdehnungscoëfficient . . . . . 0,00000095

$\begin{array}{lllllllll}\text { T'tmperatur } & 120 & 139 & 159 & 182 & 210 & 235 & 261 & 287\end{array}$

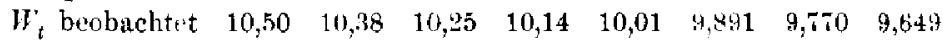

$W_{t}=11,2\left(1-0,000588 t+0,000000434 t^{2}\right)$.

Specitischer Widerstand $=952,0$.

Kohle VIII.

Künstliche Kohle Nr. $5 \tau$ bezogen von $K$ aiser \& Schmidt in Berlin.

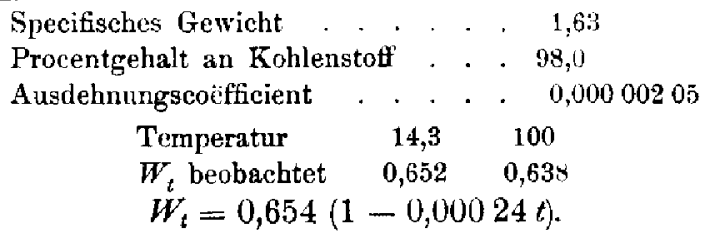

Specifischer Widerstand $=36,86$.

Kohle IX.

Eine zweite künstliche Kohle von Kaiser \& Schmidt in Berlin.

$\begin{array}{ccc}\text { Temperatur } & 11,7 & 100 \\ W_{t} \text { beobachtet } & 1,224 & 1,186 \\ W_{t}=1,231 & (1-0,00037 t) . \\ \text { Specifischer } & \text { Widerstand }=41,17 .\end{array}$

1) Kohle VI zeigte ein merkwürdiges Verhalten, welches wahrscheinlich auf Contactfehlerm berahte. Ich verzichte hier auf die Angabe dieser Messungen und verweise auf meine Dissertation. Strassburg 1881. 


\section{Kohle X.}

Künstliche Kohle für electrisches Licht ron Goudoin.

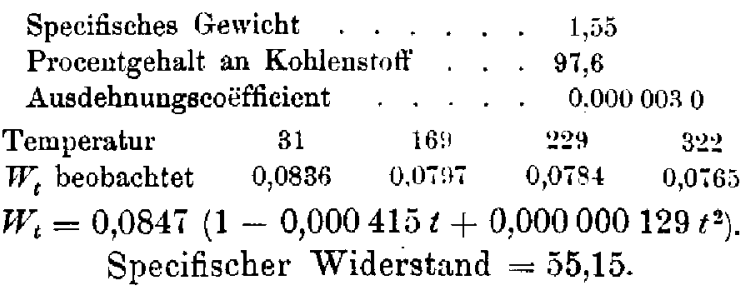

§4. Thermoelectrisches Verhalten verschiedener Koble gegeneinander.

Während der in dem bisherigen Theil der Arbeit beschriebenen Untersuchungen des Widerstandes der Kohlen bemerkte ich, dass die Thermoströme zwischen den Kohlen und den Zuleitungsdrähten sowohl, als auch zwischen zwei verschiedenen Kohlen nicht unbedeutend sind. Da möglicherweise die thermoelectromotorische Kraft in Zusammenhang mit dem Leitungswiderstand oder vielleicht mit der Aenderung desselben steht, so untersuchte ich die Ströme zwischen dem oben genannten sibirischen Graphit (Kohle I) und den verschiedenen Kohlen, indem ich die Ströme mit einem KupferNeusilberelement verglich, welches letztere seinerseits mit einem Daniell'schen Element verglichen wurde. Die Messungsmethode war die Poggendorff'sche. ${ }^{1}$ ) In den Stromkreis $x$ des zu messenden Elementes schaltete ich Drahtrollen $R$ von bekannten Widerständen mit Quecksilbercontact ein, während in dem Kreise $n$ des Normalelementes ein Siemens'scher Rheostat $R h$ sich befand, von welchem ich nur Stöpsel benutzte, da der Schiebercontact für diesen $Z$ weck wohl nicht sicher genug ist. Kleinere Widerstände als eine S.-E. konnten aus den Ablenkungen der Galvanometernadel interpolirt werden. Die Löthstellen der beiden Elemente wurden auf $100^{\circ}$ und die Enden auf $0^{\circ}$ gehalten. Steht das Galranometer auf Null für die in die beiden Stromkreise eingeschalteten Widerstände:

1) Poggendorff, Pogg. Ann. o4. p. 161. 1841. 


$$
\begin{aligned}
R & \left.=r_{x}^{\prime}, \quad r_{x}^{\prime \prime}, \quad r_{x}^{\prime \prime \prime} \ldots r_{*}^{p}, \quad r_{*}^{q} \ldots{ }^{3}\right) \\
R h & =r_{n}^{\prime}, \quad r_{n}^{\prime \prime}, \quad r_{n}^{\prime \prime \prime} \ldots r_{n}^{p}, \quad r_{n}^{q} \ldots,
\end{aligned}
$$

so ist: $E=\frac{r_{x}^{\prime}-r_{x}^{\prime \prime}}{r_{n}^{\prime}-r_{n}^{\prime \prime}}=\frac{r_{x}^{\prime \prime}-r_{x}^{\prime \prime \prime}}{r_{n}^{\prime \prime}-r_{n}^{\prime \prime \prime}}=\cdots \frac{r_{*}^{P}-r_{x}^{q}}{r_{n}^{p}-r_{n}^{q}}=\cdots$

wenn $E$ die thermoelectrische Kraft des Kohlenelementes, bezogen auf die des Normalelementes gleich 1, bedeutet. Die aus den Beobachtungen sich ergebenden Zahlen sind folgende:

\begin{tabular}{|c|c|c|c|}
\hline Nr. der Beob. & I & II & III \\
\hline$r_{x}$ & $0, \overline{5} 06$ & 1,000 & 1,506 \\
\hline$r_{n}$ & $b 5,4$ & $\pm 31,0$ & 199,0 \\
\hline Combination & $\mu_{x}^{p}-r_{x}^{q}$ & $\boldsymbol{r}_{n}^{p}-\boldsymbol{r}_{n}^{\psi}$ & $E$ \\
\hline I, II & $0,496^{\circ}$ & 65,6 & 0,00753 \\
\hline I, III & 1,000 & 133,6 & 0,00748 \\
\hline II, III & 0,506 & 68,0 & 0,00744 \\
\hline
\end{tabular}

Graphit gegen Kohle II.

\begin{tabular}{|c|c|c|c|c|c|c|c|}
\hline & & Gra & L g & n $\mathbf{K}_{0}$ & $I V$ & & \\
\hline Nr. d & 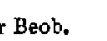 & I & II & III & IV & $T$ & \\
\hline & $x$ & 0,506 & 1,000 & 1,506 & 1,98 & & 86 \\
\hline & $n$ & 47,0 & $i 4,0$ & 105,0 & 132,0 & 192 & \\
\hline mbin. & $r_{*}^{p}-r_{*}^{q}$ & $r_{n}^{p}-r_{n}^{q}$ & $E$ & Combin. & $r_{x}^{p}-r_{x}^{q}$ & $r_{n}^{p}-r_{n}^{q}$ & $E$ \\
\hline I. II & 0,494 & $2 \pi, 0$ & 0,0183 & II, IV & $0,9 \times 6$ & 58,0 & 0,0170 \\
\hline I. III & 1,000 & 58,0 & 0,0172 & II, V & 1,986 & 118,0 & 0,016 \\
\hline I. IV & 1,480 & 85,0 & 0,0173 & III, IV & 0,408 & 27,0 & 0,0178 \\
\hline I, V & 2,480 & $14 \overline{5}, 0$ & 0,0171 & UI, V & $1, \pm 80$ & 87,0 & 0,0170 \\
\hline II, III & 0,506 & 31,0 & 0,0163 & IV, V & 1,000 & 60,0 & 0,016 \\
\hline
\end{tabular}

Mittel: Graphit - Kohle II $=-0.00748$.

Mittel: Graphit - Kohle IV $=-0,0171$.

Graphit gegen Kohle V.

$\begin{array}{cccc}\text { Sr. der Beab. } & \text { I } & \text { II } & \text { III } \\ r_{*} & 0,506 & 1,000 & 1,986 \\ r_{n} & 35,0 & 61,0 & 113,0 \\ \text { Combination } & r_{x}^{p}-r_{*}^{q} & r_{n}^{p}-r_{n}^{q} & E \\ \text { I, II } & 0,494 & 26,0 & 0,0190 \\ \text { I, IUI } & 1,480 & 78,0 & 0,0190 \\ \text { II, III } & 0,986 & 52,0 & 0,0190\end{array}$

Mittel: Graphit - Kohle $V=-0,0190$.

1) Die Indices $x$ und $n$ deuten die Stromkreise des Kohle-, resp. Normalelementes an. 
Graphit gegen Kohle VII.

\begin{tabular}{|c|c|c|c|c|c|c|c|}
\hline & $\begin{array}{l}\text { er Beob. } \\
r_{x} \\
r_{n}\end{array}$ & $\begin{array}{c}I \\
1,000 \\
22,0\end{array}$ & $\begin{array}{c}\text { II } \\
2,986 \\
27,0\end{array}$ & $\begin{array}{c}\text { III } \\
3,962 \\
30,0\end{array}$ & $\begin{array}{c}\text { IV } \\
10,08 \\
49,5\end{array}$ & $\begin{array}{r}\mathbf{v} \\
45,6 \\
157,0\end{array}$ & \\
\hline Combin. & $r_{n}^{p}-r_{n}^{p}$ & $r_{n}^{p}-r_{n}^{q}$ & $E$ & Combin & $r_{x}^{p}-r_{*}^{q}$ & $r_{n}^{p}-r_{n}^{q}$ & $E$ \\
\hline I, II & 1,986 & 5,0 & 0,397 & II, IV & 7,094 & 22,5 & 0,3 \\
\hline I, III & 2,962 & 8,0 & 0,370 & II, V & 42,624 & 130,0 & 0,320 \\
\hline I, IV & 9,080 & 27,5 & 0,330 & III, IV & 6,118 & 19,5 & 0,314 \\
\hline $\mathbf{I}, \mathbf{V}$ & 44,610 & 135,0 & 0,330 & III, V & 41,650 & 127,0 & 0,328 \\
\hline II, III & 0,976 & 3,0 & 0,325 & $\mathrm{IV}, \mathrm{V}$ & 35,530 & 107,0 & 0,332 \\
\hline
\end{tabular}

Graphit gegen Kohle VIII.

\begin{tabular}{|c|c|c|c|c|c|c|c|}
\hline & $\begin{array}{l}\text { ler Beob. } \\
r_{n} \\
r_{n}\end{array}$ & $\begin{array}{c}\text { I } \\
0,506 \\
28,0\end{array}$ & $\begin{array}{c}\text { II } \\
1,000 \\
41,0\end{array}$ & $\begin{array}{c}\text { III } \\
1,506 \\
5 \overline{5}, 0\end{array}$ & $\begin{array}{c}\text { IV } \\
1,986 \\
67,0\end{array}$ & $\begin{array}{c}V \\
2,98 \\
95,0\end{array}$ & \\
\hline mbin. & $r_{x}^{p}-r_{x}^{q}$ & $r_{n}^{p}-r_{n}^{q}$ & $E$ & Combin. & $r_{x}^{p}-r_{x}^{q}$ & $r_{n}^{p}-r_{n}^{q}$ & $E$ \\
\hline I. I & 0,494 & 13,0 & 0,0380 & II, IV & 0,986 & 26,0 & 0,0379 \\
\hline I, III & 1,000 & 27,0 & 0,0370 & II, V & 1,986 & 54 , & 0,0368 \\
\hline I, IV & 1,480 & 39,0 & 0,0380 & III, IV & 0,480 & 12,0 & 0,0400 \\
\hline $\mathrm{I}, \mathrm{V}$ & 2,480 & 67,0 & 0,0370 & III, V & 1,480 & 40,0 & 0,0370 \\
\hline I, III & 0,506 & 14,0 & 0,0361 & IV, V & 1,000 & 28,0 & 0,0357 \\
\hline
\end{tabular}

Graphit gegen Kohle X.

$\begin{array}{ccccc}\text { Nr. der Beob. } & \text { I } & \text { II } & \text { III } & \text { IV } \\ r_{*} & 1,000 & 1,506 & 1,986 & 2,986 \\ r_{n} & 20,0 & 29,9 & 39,0 & 59,0\end{array}$

\begin{tabular}{llcc|cccc} 
Combin. & $r_{x}^{p}-r_{x}^{q}$ & $r_{n}^{p}-r_{n}^{q}$ & $E$ & Combin. & $r_{x}^{p}-r_{x}^{q}$ & $r_{n}^{p}-r_{n}^{q}$ & $E$ \\
I, II & 0,506 & 9,9 & 0,0511 & II, III & $\mathbf{0 , 4 8 0}$ & 9,1 & 0,0527 \\
I, III & $\mathbf{0 , 9 8 6}$ & $\mathbf{1 9 , 0}$ & $\mathbf{0 , 0 5 1 9}$ & II, IV & $\mathbf{1 , 4 8 0}$ & $\mathbf{2 9 , 1}$ & $\mathbf{0 , 0 5 0 9}$ \\
I, IV & $\mathbf{1 , 9 8 6}$ & $\mathbf{3 9 , 0}$ & $\mathbf{0 , 0 5 0 9}$ & III, IV & $\mathbf{1 , 0 0 0}$ & $\mathbf{2 0 , 0}$ & $\mathbf{0 , 0 5 0 0}$
\end{tabular} Mittel: Graphit - Kohle X $=-0,0513$.

Graphit gegen Kohle VI.

Bei diesem Element war der Widerstand der Kohle allein schon so gross, dass bei ihrer electromotorischen Kraft der Ausschlag des Spiegels sehr klein wurde. Ich konnte nicht noch neue Widerstände einschalten, sondern musste 
die electromotorische Kraft aus den Gesammtwiderständen in den beiden Stromkreisen $x$ und $n\left(W_{x}=2,057, W_{n}=46,663\right)$ berechnen. Es ergab sich dann:

$$
\text { Graphit - Kohle VI }=-0,0190 \text {. }
$$

Zur Vergleichung des Normalelementes mit einem Daniell bediente ich mich ebenfalls der Poggendorff'schen Methode. Die Anordnung war dieselbe wie oben, nur musste im Zweige des Daniell'schen Elementes ausser dem Siemens'schen Widerstandskasten noch ein grosser Widerstand eingeschaltet werden, damit die Beobachtung überhaupt möglich war. Diesen $W$ iderstand brauchte ich natiirlich nicht zu kennen. Als Flüssigkeiten für das Daniell'sche Element nahm ich concentrirte Kupfervitriollösung und verdünnte Schwefelsäure (1 Vol. $\mathrm{H}_{2} \mathrm{SO}_{4}$ und $8 \mathrm{Vol} . \mathrm{H}_{3} \mathrm{O}$ ). In der folgenden Angabe bedeutet $r_{d}$, dem $r_{x}$ entsprechend; den Widerstand, der in den Stromkreis des Daniell'schen Elementes eingeschaltet wurde.

Kupfer-Neusilberelement.

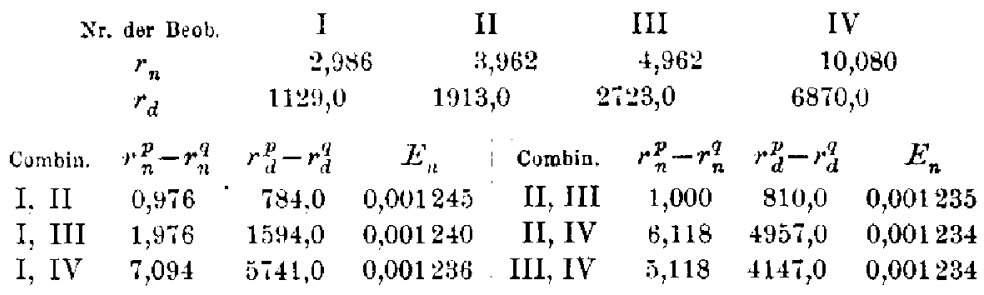

Mittel: Kupfer-Neusilberelement $=+0,00124 \mathrm{D}$.

Becquerel ${ }^{1}$ ) fand für das Kupfer-Neusilberelement den Werth $E=0,00126$ D., ein Werth, der mit der obigen Zahl genügend übereinstimmt. Setzt man 1 Daniell gleich 10000000 , so kommt als Resultat meiner Messung Folgendes:

Electromotorische Kraft $E$ der Thermoströme zwischen Kohlen und Graphit (Kohle I) ausgedruckt in Zehnmillionteln eines Daniells.

\begin{tabular}{l|c|c|c|ccc|cc}
\hline Nr. der Koble & I & II & IV & V & VI & VII & VIII & X \\
\hline$E$ & \pm 0 & $+92,6$ & +213 & +235 & +546 & +4230 & +464 & +635
\end{tabular}

1) Becquerel, Ann. de chim. et de phys. (4) 7. p. 415.1864. 
Die einzelnen Beobachtungen der Thermoströme der Kohlen stimmen nicht so gut überein, wie die bei der Vergleichung des Normalelementes mit dem des Daniells. Es kann dies in der Ungenauigkeit der Interpolation liegen, möglicherweise aber auch darin, dass ein Kohlenelement nicht so constant ist wie ein Metallelement.

\section{§. Z. Zusammenstellung der Resultate.}

Zur besseren Uebersicht stelle ich hier die Resultate zusammen, indem ich die Kohle nach der Grösse des specitischen Widerstandes ordne.

\begin{tabular}{|c|c|c|c|c|c|c|c|}
\hline $\begin{array}{c}\text { Sr. } \\
\text { der } \\
\text { huble }\end{array}$ & 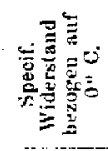 & $\begin{array}{c}\text { Temperatur- } \\
\text { cuéfficient } \\
\text { a }\end{array}$ & $\begin{array}{c}\text { Temperatur- } \\
\text { coefficientt } \\
\beta\end{array}$ & 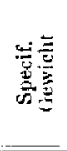 & 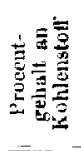 & 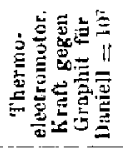 & 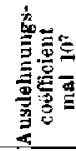 \\
\hline VII & 952.0 & $-0,000$.58 & $+0,000000+34$ & 2,36 & 52.0 & +4230 & 0,5 \\
\hline $\mathrm{X}$ & 55,15 & $-0,000+15$ & $+0,0000100012 ! !$ & 1.55 & 97.6 & +635 & 30 \\
\hline II & 52,23 & $-0,000300$ & $-\cdots$ & 1,80 & - & $+\quad 92,6$ & 15 \\
\hline III & $4 \times, 44$ & $-0,000405$ & - & - & - & - & - \\
\hline IV & 42,90 & $-0,000+25$ & ד, & 2.37 & - & +213 & 15 \\
\hline IX & 41.17 & $-0,000370$ & - & - & - & 一 & - \\
\hline $\mathrm{V}$ & 39,10 & $-0,000156$ & $+0,000000524$ & 1,90 & 98,1 & +235 & 3,2 \\
\hline VIII & 36,86 & $-0,000) \cdot 40$ & - & 1,63 & 98,0 & $+4 B t$ & 90.5 \\
\hline I & $1 \geq .20$ & $-0,000739$ & $+0,000000273$ & 1,80 & 98,0 & \pm 0 & 38 \\
\hline
\end{tabular}

Die in der Tabelle angegebenen physikalischen Eigenschuften lassen keine regelmässigen Beziehungen zum Widerstand oder zu dessen Aenderung erkennen. Es lässt sich ja auch von vornherein vermuthen, dass die Grösse der Widerstände oder deren Aenderungen nicht in einer einfachen Beziehung zu den anderen physikalischen Eigenschaften stehen, sondern, dass sie eine sehr complicirte Function dieser Eigenschaften sind, weshalb Schrader ${ }^{1}$ ) auch keine Beziehung zwischen den Widerständen und den specifischen Gewichten fand. Unter den verschiedenen Kohlen ist der Graphit am charakteristischsten; er hat den kleinsten galvanischen Widerstand, die grösste Aenderung des Widerstandes mit der Temperatur, und er ist electronegativ

1) Schrader, Gött. Nachr. p. 325. 1875. 
gegen alle anderen Kohlen, er scheint ausserdem der beste Wärmeleiter zu sein, was ich aber leider nicht quantitativ untersuchen konnte. Der Graphit zeichnet sich aber auffallenderweise nicht durch den Procentgehalt an Kohlenstoff und durch das specifische Gewicht aus.

Was die Hauptfrage, die Aenderung des Widerstandes mit der Temperatur, anbetrifft, so ist festgestellt, dass der Widerstand bei höherer Temperatur abnimmt, und zwar bei allen untersuchten Sorten ron Kohlen.

Endlich ist noch zu erwähnen, dass Holzkohlen und Steinkohlen, welche ich untersuchte, mit dem von mir benutzten Galvanometer keine Leitung erkennen liessen.

Phys. Inst. der Univ. Strassburg, März 1881.

VIII. Bemerkungen zu der Abhandlung von Hrn. Warburg: ,Ueber einige Wirkungen der magnetischen Coërcitivliraft; von Ca»l Fromme. (Aus den Gött. Nachr. vom Februar 1881 mitgetheilt vom Hrn. Verf.)

Die von Hrn. Warburg mitgetheilten Untersuchungen 1) stehen zu den Arbeiten, welche ich vor einigen Jahren ${ }^{9}$ ) unter dem Titel "Magnetische Experimentaluntersuchungen" veröffentlicht habe, in so naher Beziehung, dass ich mir wohl erlauben darf, einige Bemerkungen an dieselben zu knüpfen.

Die ron mir mitgetheilten Versuche ${ }^{3}$ ) bestanden darin, dass ich einen Stahlstab, dessen permanenter Magnetismus nahe seinen Maximalwerth besass, sodass er durch die angewandten Kräfte nicht mebr gesteigert wurde, der magnetisirenden Kraft $\boldsymbol{P}$ eines Stromes von zwei Bunsen unterwarf. dessen Intensität infolge der gewählten, sehr verdünnten Salpetersäure stark abnahm.

1) Warburg, Wied. Ann. 13. p. 14l-164. 1881.

2) Fromme, Wied. Ann. 4. p. $76-107.1878 .5$. p. 345-388. 1878.

3) Fromme, Wied. Ann. 4. p. 102-105. 1878. 